\title{
Regional density of glial cells in the rat corpus callosum
}

\author{
Daniel Reyes-Haro*,‡, Ernesto Mora-Loyola ${ }^{\ddagger}$, Berenice Soria-Ortiz and Jesús García-Colunga
}

Departamento de Neurobiología Molecular y Celular. Instituto de Neurobiología, Universidad Nacional Autónoma de México, Campus Juriquilla, Boulevard Juriquilla 3001 Juriquilla, Querétaro CP 76230, México.

\begin{abstract}
Axons and glial cells are the main components of white matter. The corpus callosum (CC) is the largest white matter tract in mammals; in rodents, $99 \%$ of the cells correspond to glia after postnatal day 5 (P5). The area of the CC varies through life and regional differences related to the number of axons have been previously described. Whether glial cell density varies accordingly is unknown; thus the aim of this study was to estimate glial cell density for the genu, body and splenium -the three main regions of CC-, of P6 and P30 rats. Here we report that the density of CC glial cells reduced by $\sim 10 \%$ from P6 to P30. Even so, the density of astrocytes showed a slight increase ( $+6 \%)$, probably due to differentiation of glioblasts. Interestingly, glial cell density decreased for the genu (-21\%) and the body (-13\%), while for the splenium a minor increase $(+5 \%)$ was observed. The astrocyte/glia ratio increased (from P6 to P30) for the genu $(+27 \%)$, body $(+17 \%)$ and splenium $(+4 \%)$. Together, our results showed regional differences in glial cell density of the CC. Whether this pattern is modified in some neuropathologies remains to be explored.
\end{abstract}

Key words: Astrocytes, corpus callosum, genu, glial cells, splenium

\section{INTRODUCTION}

The corpus callosum (CC) is the main white matter tract in the brain and is involved in interhemispheric communication. This commissure is composed of axons and glial cells, while neuronal somata make up less than $1 \%$. After postnatal day 5 (P5), glial cells predominate, accounting for $99 \%$ of all cells (Sturrock, 1976; Aboitiz \& Montiel, 2003). The size, myelination, and density of fibers in callosal subregions are related to the brain regions they connect (Doron \& Gazzaniga, 2008). Thus thin unmyelinated fibers are most dense in the genu and splenium (anterior and posterior CC), where they connect association and prefrontal areas, while large diameter myelinated fibers are concentrated in the midbody of the $\mathrm{CC}$, where they connect primary and secondary sensory and motor areas (Aboitiz, 1992). Interestingly, electron microscopic analysis revealed that the number of callosal axons increases from 4.4 million at birth to 11.4 million at P5 and this number is maintained until the adult stage in rats (P60) (Gravel et al., 1990), although the number of axons of the visual cortex projecting through the splenium of the rat CC decreased by 15\% from P15 to P60 (Kim \& Juraska, 1997). On the other hand, myelination in the CC starts with non-compacted cytoplasmic sheaths of myelin at P12, and each oligodendrocyte sheathes an average of 13 axons. Later, when the compact myelin is formed, a single oligodendrocyte sheathes, on average, 15 axons (P17) (Bjartmar et al., 1994). Considering all this information, CC regions might differ in glial cell density. Interestingly, it is not known if the glial cell density increases or remains constant through development, or if the distribution of glial cells and the astrocyte/glial cell ratios are different among different regions of the CC. Therefore, the aim of this study was to estimate glial cell densities in the CC at two ages (P6 and P30) and to determine if there are differences among callosal regions.

\section{MATERIAL AND METHODS}

Histology

Six female rats per age group (P6 and P30) were anesthetized with an overdose of sodium pentobarbital $(100 \mathrm{mg} / \mathrm{Kg})$ and then decapitated. Whole brains were removed rapidly and dissected on ice. The hemispheres were separated and fixed with $4 \%$ paraformaldehyde in phosphate buffered saline (PBS, $0.1 \mathrm{M}, \mathrm{pH} 7.4$ ) for $24 \mathrm{~h}$ and then transferred to $30 \%$ sucrose in $0.1 \mathrm{M}$ PBS at $4{ }^{\circ} \mathrm{C}$ for cryoprotection. Sagittal sections $(30 \mu \mathrm{m}$; two consecutive from the right hemisphere and one the from left hemisphere $=3$ slides per animal) were obtained using a freezing cryostat and collected on gelatinized slides for staining with propidium red to estimate glial cell number (Fig. 1 and 2).

Immunohistofluorescence

Cryosections were blocked in PBS containing 1\% bovine serum albumin, $1 \%$ goat serum, and $0.1 \%$ Triton $\mathrm{X}-100$ for 1 $\mathrm{h}$ and labeled overnight at $4{ }^{\circ} \mathrm{C}$ with a 1:500 dilution of antiglial fibrillary acidic protein (monoclonal mouse anti-GFAP, Milipore, USA). After incubation with the primary antibody, samples were rinsed 3 times in PBS and labeled for $1 \mathrm{~h}$ at room temperature with a 1:500 dilution of Alexa 488 goat antimouse antibody (Invitrogen, USA). Finally, slides with sagittal sections containing the CC were washed 3 times with PBS, stained with propidium red and mounted with Vectashield (Vector laboratories, Inc, USA). Slides were examined with an epifluorescence microscope (Olympus BX60) coupled to a digital camera (Olympus DP70). Image-Pro MC for Windows was used for estimation of glial cell density. A Zeiss LSM 510 Meta confocal microscope (Zeiss, Göttingen, Germany) 
was used for confocal images, with wavelength $488 \mathrm{~nm}$ for excitation of Alexa 488 and propidium red. The emission wavelengths were $519 \mathrm{~nm}$ and $610 \mathrm{~nm}$ respectively. The z-stack images (4 to 5 consecutive confocal sections of $512 \times 512$ ) were obtained every $5 \mu \mathrm{m}$ with stack size of X: $450 \mu \mathrm{m}$ and Y: $450 \mu \mathrm{m}$ and processed in Aim Image Examiner.

\section{Glial cell counting}

The population of cells immunostained by the GFAP antibody was contrasted with the number of propidium red-labeled nuclei for each slide and each CC region. Data analysis (counting) was performed using Image J software $1.41(\mathrm{NIH}$, National Institute of Health, Bethesda, Maryland, USA).

A test square grid of $0.01 \mathrm{~mm}^{2}$ in each section was used to estimate the number of propidium red-stained cell nuclei equivalent to the total number of glial cells including $\mathrm{GFAP}^{+}$ cells corresponding to astrocytes (only process-bearing cells showing their soma in the plane of the section were counted). Six randomly chosen fields (as indicated in Figs. 1D and 2D) were counted by progressive displacement of the test square grid. The astrocyte / glial cell ratio results from the estimation of the GFAP+ cells divided by the total number of glial cells labeled with propidium red. Data are presented as the mean \pm standard error of the mean (S.E.M.) and nonparametric statistics were performed with the Mann-Whitnney-U test; $\mathrm{p}<$ 0.05 was considered significant.

\section{RESULTS}

\section{The pool of glial cells is reduced from P6 to P30}

Our estimations of glial cell density began at P6 because the nuclear counts could be related to the estimation of glial cell number (99\% after P5; Sturrock, 1976); P30 (considered as an adult stage) was selected for comparison. We found that the number of glial cells decreased from $1880 \pm 45 / \mathrm{mm}^{2}$ (P6) to $1672 \pm 27 / \mathrm{mm}^{2}$ (P30) ( $\mathrm{p}=0.00012$, Fig. 3). On the other hand, the number of astrocytes $\left(\mathrm{GFAP}^{+}\right.$cells) showed a $+7 \%$ increase, from $135 \pm 1.4 / \mathrm{mm}^{2}$ at P6 to $144 \pm 1.59 / \mathrm{mm}^{2}$ at P30 (p< 0.0001 ; Fig. 3 ). These results indicate that the pool of glial cells of CC is reduced and that a fraction of glioblasts differentiated into astrocytes between P6 and P30.
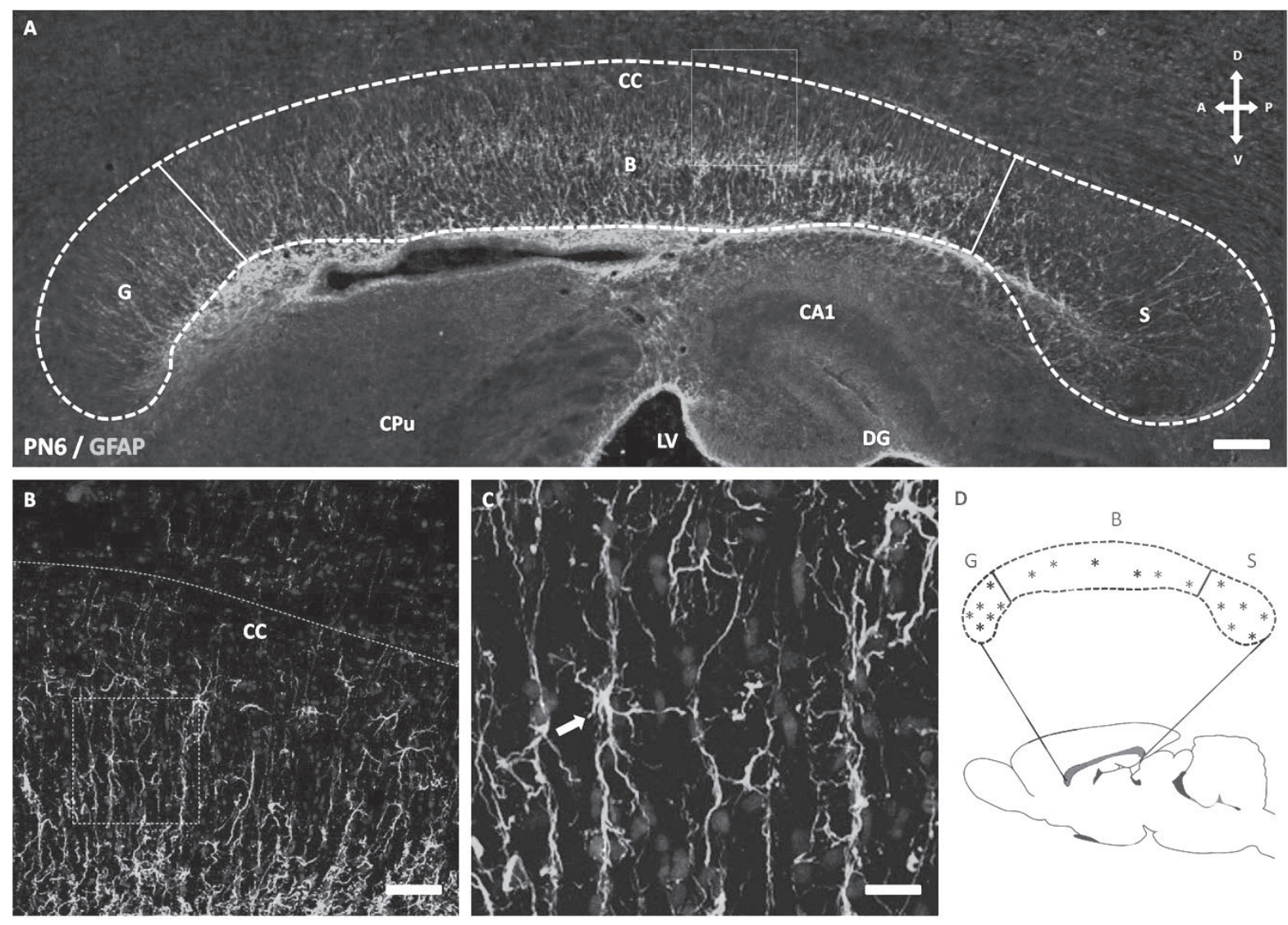

Figure 1. The rat corpus callosum (CC) at P6. A, Sagittal sections of the rat brain were immunostained for GFAP (18 slides from 6 brains; 3 per brain) and propidium red. The CC was dissected in 3 regions: genu (G), body (B) and splenium (S) for estimation of glial cell density $(n=18$ per region). Other brain regions are indicated for anatomical reference: hippocampal CA1 region (CA1), caudate-putamen (CPu), hippocampal dentate gyrus (DG), lateral ventricle (LV). Arrows showing anatomical orientation: dorsal (D), ventral (V), anterior (A) and posterior (P). B, Zoom of the square in A to show astrocytes from CC, notice the radial projections of astrocytes. C, Zoom of the square in B to highlight radial glia (arrow). Scale bars $200 \mu \mathrm{m}$ (A), $80 \mu \mathrm{m}$ (B), $20 \mu \mathrm{m}$ (C). D, The representative sites (*) used for estimation of glial cell density are highlighted in a diagram of CC. Three slides containing sagittal sections of CC where used for each brain and each region of CC included 2 selected sites per slide for estimations (6 sites per region, per rat brain). 



Figure 2. The rat corpus callosum (CC) at P30. $A$, Sagittal sections of the rat brain were immunostained for GFAP and propidium red (18 slides from 6 brains; 3 per brain). The CC was dissected in 3 regions: genu (G), body (B) and splenium (S) for estimation of glial cell density ( $n=18$ per region). Other brain regions are indicated for anatomical reference: hippocampal $\mathrm{CA} 1$ region $(\mathrm{CA} 1)$, caudate-putamen $(\mathrm{CPu})$, hippocampal dentate gyrus (DG), lateral ventricle (LV). Arrows showing anatomical orientation: dorsal (D), ventral (V), anterior (A) and posterior (P). B, Zoom of the square in $A$ to show fibrous astrocytes from $\mathrm{CC}$, notice that radial projections of astrocytes observed at P6 are absent at P30. C, Zoom of the square in B to highlight fibrous astrocytes (arrow). Scale bars $250 \mu \mathrm{m}$ (A), $80 \mu \mathrm{m}$ (B), $20 \mu \mathrm{m}$ (C). D, The representative sites $(*)$ used for estimation of glial cell density are highlighted in a diagram of CC. Three slides containing sagittal sections of CC where used for each brain and each region of CC included 2 selected sites per slide for estimations (6 sites per region, per rat brain).

Glial cells are more abundant in the genu

The size, myelination, and density of fibers in callosal subregions are related to the brain regions they connect (Doron \& Gazzaniga, 2008). We estimated glial cell density to be greater in the genu at P6 $\left(2319 \pm 32 / \mathrm{mm}^{2}\right)$ than in the body or splenium (1616 $\pm 14 / \mathrm{mm}^{2}$ and $1704 \pm 14 / \mathrm{mm}^{2}$, respectively; $\mathrm{p}<0.0003$ ) (Fig. 4A). A similar pattern was observed at P30, but here glial cell density of the splenium was closer to that of the genu; glial cell density in the genu was $1824 \pm 8$ / $\mathrm{mm}^{2}$, whereas for the body and splenium there were $1405 \pm 9$ and $1788 \pm 13$ cells $/ \mathrm{mm}^{2}$, respectively $(\mathrm{p}<0.001)$ (Fig. 4B). Therefore, we found a higher density of glial cells in the genu than in the body or splenium, at both P6 and P30.
A
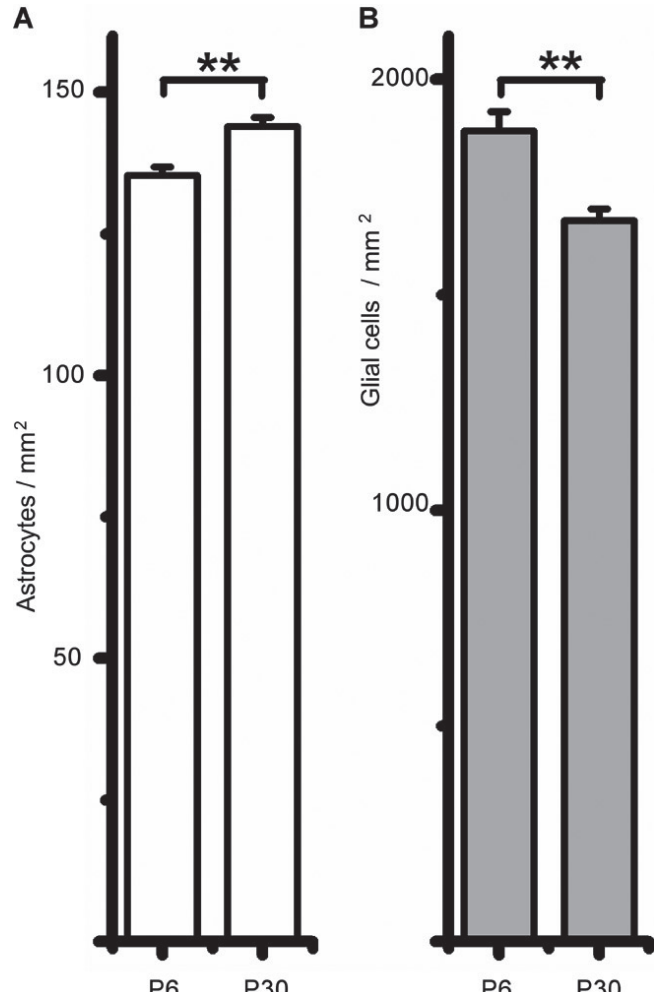

Figure 3. Estimation of glial cell density for rat CC at P6 and P30. $A$, The number of astrocytes per area $(\mathrm{mm} 2)$ increased from P6 $(n=52)$ to P30 ( $n=53)$. B, The number of glial cells decreased from P6 $(n=53)$ to P30 $(n=54)$. The estimation included all the regions of rat CC. Data are mean \pm S.E.M. $(* * p<0.001)$. 
The glial cell density reduces from P6 to P30 in the genu and body

Our next step was to compare regional glial cell density between P6 and P30. The results showed that glial cell density was reduced in the genu (-21\%; from $2319 \pm 32 / \mathrm{mm}^{2}$ (P6) to $1824 \pm$ $\left.8 / \mathrm{mm}^{2}(\mathrm{P} 30) ; \mathrm{p}<0.0001\right)$ and in the body $(-18 \%$; from $1616 \pm 14$ / $\mathrm{mm}^{2}$ (P6) to $\left.1405 \pm 9 / \mathrm{mm}^{2}(\mathrm{P} 30) ; \mathrm{p}<0.0001\right)$. Interestingly, the splenium showed a different pattern because a slight increase in glial cell density was observed (+5\%; from $1704 \pm 14 / \mathrm{mm}^{2}(\mathrm{P} 6)$ to $1788 \pm 13 / \mathrm{mm}^{2}$ (P30); $\left.\mathrm{p}=0.001\right)$ (Table 1$)$.

Astrocyte density increases from P6 to P30

Astrocytes increased in number in the genu (+6.8\%; from 147 $\pm 2 / \mathrm{mm}^{2}$ (P6) to $\left.157 \pm 2 / \mathrm{mm}^{2}(\mathrm{P} 30) ; \mathrm{p}=0.006\right)$, the body $\left(+4.6 \%\right.$; from $128 \pm 1 / \mathrm{mm}^{2}(\mathrm{P} 6)$ to $134 \pm 2 / \mathrm{mm}^{2}$ (P30); $\mathrm{p}=0.0209)$ and the splenium $\left(+9.2 \%\right.$; from $130 \pm 1 / \mathrm{mm}^{2}(\mathrm{P} 6)$ to $142 \pm 2 / \mathrm{mm}^{2}$ (P30); $\left.<<0.0001\right)$ (Table 1).

We then estimated the astrocyte / glia ratio at P6 and P30, based on GFAP immunoreactivity and nuclear staining. We consider that this ratio is a more accurate estimation because it considers the glial cell density, which varies among CC regions. Our estimations showed that the astrocyte / glia ratio increased by $+27 \%$ for the genu (from $0.0637 \pm 0.0014$ to 0.0858 $\pm 0.0008 ; \mathrm{p}<0.0001),+17 \%$ for the body (from $0.0794 \pm 0.0008$ to $0.0951 \pm 0.0014 ; \mathrm{p}<0.0001$ ) and $+4 \%$ for the splenium (from $0.0766 \pm 0.0008$ to $0.0793 \pm 0.0008 ; \mathrm{p}=0.066$ ) (Fig. 5). Overall, these results indicate that the density of astrocytes increases during postnatal development, because a fraction of glioblasts differentiate to astrocytes before P30.

\section{DISCUSSION}

The development of the CC is not homogenous through its three main regions; the genu, body, and splenium. Different changes in callosal thickness occur due to axonal myelination, redirection, and pruning (LaMantia \& Rakic, 1990; Luders et al., 2010). Nevertheless, glial cell density has not been explored among CC regions during postnatal development. Here, we report that the pool of glial cells decreased from P6 to P30. During this period of development, glioblasts (the dominant cell type in CC at P5) differentiate mainly to oligodendrocytes and astrocytes (Mori \& Leblond, 1970; Sturrock, 1976). Surprisingly, when the glial cell density was estimated in the three regions of the rat $\mathrm{CC}$, we found that glial cell density was greater in the genu at both P6 and P30 than in the body or the splenium. The functional significance of the greater density of glial cells in the genu remains to be investigated.
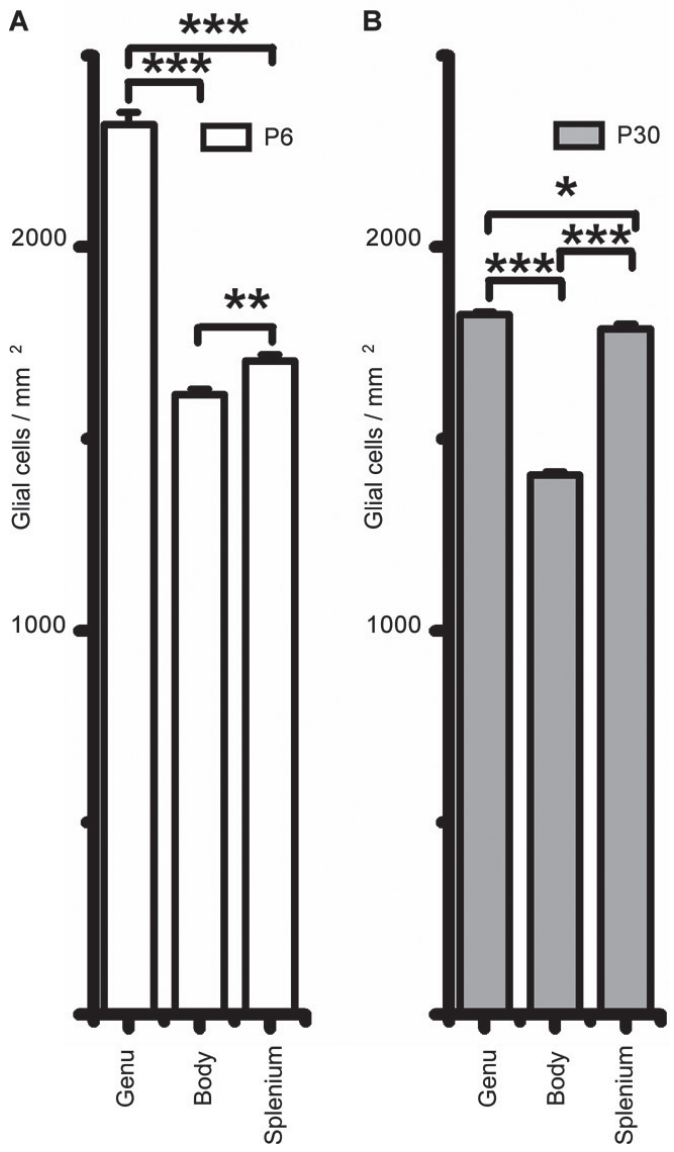

Figure 4. Regional glial cell density in rat CC at P6 and P30. The genu showed the highest density of glial cells among regions, at P6 $(A ; n=17$ per region) and $P 30(B ; n=18$ per region). Data are mean \pm S.E.M. ( ${ }^{* * *} p<0.0001$; $\left.^{* *} \mathrm{p}<0.001{ }^{*} \mathrm{p}<0.05\right)$.

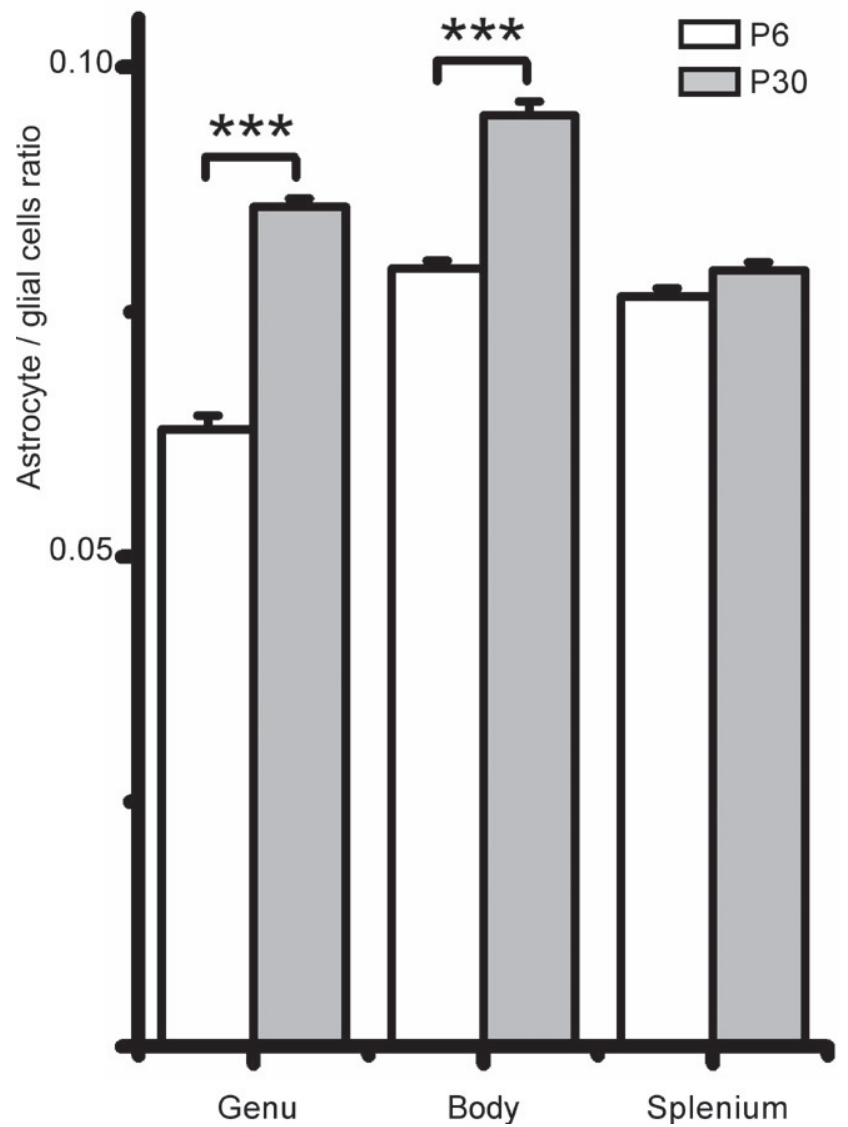

Figure 5. Astrocyte / glial cell ratio in rat $C C$ at P6 and P30. The proportion of astrocytes among glial cells increased significantly for the genu $(n=17)$ and the body $(n=18)$ (P6 and P30), but not for the splenium ( $n=17$ (P6) and $n=18$ (P30)). Data are mean \pm S.E.M. $(* * *)<0.0001)$. 
TABLE 1

Regional changes in glial cell density from P6 to P30

\begin{tabular}{|c|c|c|c|c|}
\hline \multicolumn{5}{|c|}{ Astrocytes } \\
\hline Genu & $147 \pm 2 / \mathrm{mm}^{2}(\mathrm{n}=18)$ & $\begin{array}{c}157 \pm 2 / \mathrm{mm}^{2} \\
(\mathrm{n}=17)\end{array}$ & $+6.80 \%$ & $\mathrm{p}=0.006$ \\
\hline Body & $\begin{array}{c}128 \pm 1 / \mathrm{mm}^{2} \\
(\mathrm{n}=17)\end{array}$ & $\begin{array}{c}134 \pm 2 / \mathrm{mm}^{2} \\
(\mathrm{n}=18)\end{array}$ & $+4.60 \%$ & $p=0.0209$ \\
\hline \multicolumn{5}{|c|}{ Glial cells } \\
\hline Region of CC & P6 & P30 & $\%$ of change & $\mathrm{p}$ \\
\hline Genu & $\begin{array}{c}2319 \pm 32 / \mathrm{mm}^{2} \\
(\mathrm{n}=17)\end{array}$ & $\begin{array}{c}1824 \pm 8 / \mathrm{mm}^{2} \\
(\mathrm{n}=18)\end{array}$ & $-21.34 \%$ & $\mathrm{p}<0.0001$ \\
\hline Splenium & $\begin{array}{c}1704 \pm 14 / \mathrm{mm}^{2} \\
(\mathrm{n}=17)\end{array}$ & $\begin{array}{c}1788 \pm 13 / \mathrm{mm}^{2} \\
(\mathrm{n}=18)\end{array}$ & $+4.92 \%$ & $\mathrm{p}=0.01$ \\
\hline
\end{tabular}

We found that the genu had the lowest astrocyte/glia ratio, with the body and splenium having similar but larger ratios at P6. However, the splenium had the lowest ratio while the body had the highest ratio at P30. These results support the hypothesis that glial cell density varies among regions of CC. Our estimations can be compared with other studies, for example, the density of astrocytes (based on $\mathrm{GFAP}^{+}$cells) that we calculated for the CC (144 cells $/ \mathrm{mm}^{2}$; P30) is similar to that in other brain regions where astrocyte markers such as GFAP or S100 $\beta$ have been used (130 astrocytes $/ \mathrm{mm}^{2}$ and 160 astrocytes $/ \mathrm{mm}^{2}$ ) (Morgan et al., 1995; Ren et al., 1992). Our estimate of astrocytes (6-9\% at P30) is slightly lower than that reported in the $\mathrm{CC}$ of rodents using morphological identification (13-15\% at P25-P45) (Sturrock, 1976), which might be due to some astrocytes that express low levels of GFAP (Zhou et al., 2000). Accordingly, the astrocyte density estimated in different brain regions was lower using GFAP than S100ß (Savchenko et al., 2000).

Overall, our results show a regional difference in glial cell density that could be related to changes in the size of the CC and the number of callosal axons (Berbel and Innocenti, 1988). This pattern could be modified when glial cell dysfunction occurs and white matter integrity is compromised (Tkachev et al.,2003; Benedetti et al., 2011). Thus, glial cell density could be affected in specific regions of the CC in some neuropathologies (Lyoo et al., 2002; Di Paola et al., 2010).

In conclusion, the glial cell density of the CC decreased while astrocyte density increased between P6 and P30. This pattern was conserved among the three regions of $\mathrm{CC}$, except for the splenium where the glial cell density increased. The genu displayed the highest density of glial cells, including astrocytes. Further studies are needed to see if the density of glial cells in the CC is differentially affected in neuropathologies such as bipolar depression or schizophrenia.

\section{AKNOWLEDGEMENTS}

We are grateful to L. González-Santos, M. García-Servín, E. Ruiz-Alcibar, E. N. Hernández-Ríos for technical support, and to Dr. Dorothy Pless for reviewing the manuscript. We are in debt to Prof. R. Miledi and Dr. A. Martínez-Torres for scientific support. This work was supported by Grants from the Consejo Nacional de Ciencia y Tecnología (CONACyT) 81911 and 166964 (to JGC and DRH); from Programa de Apoyo a Proyectos de Investigación e Innovación Tecnológica de la Universidad Nacional Autónoma de México (PAPIIT-UNAM) IN204809 and IB200412 (to JGC and DRH).

\section{REFERENCES}

ABOITIZ F (1992) Brain connections: interhemispheric fiber systems and anatomical brain asymmetries in humans. Biol Res 25: 51-61.

ABOITIZ F, MONTIEL J (2003) One hundred million years of interhemispheric communication: the history of the corpus callosum. Braz J Med Biol Res 36: 409-420.

BENEDETTI F, YEH PH, BELLANI M, RADAELLI D, NICOLETTI MA, POLETTI S, FALINI A, DALLASPEZIA S, COLOMBO C, SCOTTI G, SMERALDI E, SOARES JC, BRAMBILLA P (2011) Disruption of white matter integrity in bipolar depression as possible structural marker of illness. Biol Psychiatry 69: 309-317.

BJARTMAR C, HILDEBRAND C, LOINDER K (1994) Morphological heterogeneity of rat oligodendrocytes: electron microscopic studies on serial sections. Glia 11: 235-244.

BERBEL P, INNOCENTI GM (1988) The development of the corpus callosum in cats: a light and electron-microscopic study. J Comp Neurol 276: 132156.

DI PAOLA M, SPALLETA G, CALTAGIRONE C (2010) In vivo structural neuroanatomy of corpus callosum in Alzheimer's disease and mild cognitive impairment using different MRI techniques: a review. J Alzheimers Dis 20: 67-95.

DORON KW, GAZZANIGA MS (2008) Neuroimaging techniques offer new perspectives on callosal transfer and interhemispheric communication. Cortex 44: 1023-1029. 
GRAVEL C, SASSEVILLE R, HAWKES R (1990) Maturation of the corpus callosum of the rat: II. Influence of thyroid hormones on the number and maturation of axons. J Comp Neurol 291: 147-161.

KIM JH, JURASKA JM (1997) Sex differences in the development of axon number in the splenium of the rat corpus callosum from postnatal day 15 through 60. Brain Res Dev Brain Res 102: 77-85.

LAMANTIA AS, RAKIC P (1990) Axon overproduction and elimination in the corpus callosum of the developing rhesus monkey. J Neursoci 10: 2156-2175.

LUDERS E, THOMPSON PM, TOGA AW (2010) The development of the corpus callosum in the healthy human brain. J Neurosci 30: 10985-10990.

LYOO IK, KWON JS, LEE SJ, HAN MH, CHANG CG, SEO CS, LEE SI, RENSHAW PF (2002) Decrease in genu of the corpus callosum in medication-naive, early-onset dysthymia and depressive personality disorder. Biol Psychiatry 52: 134-143.

MORI S, LEBLOND CP (1970) Electron microscopic identification of three classes of oligodendrocytes and a preliminary study of their proliferative activity in the corpus callosum of young rats. J Comp Neurol 139: 1-28.

MORGAN TE, LAPING NJ, ROZOVSKY T, ODA T, HOGAN TH, FINCH CE, PASINETTI GM (1995) Clusterin expression by astrocytes is influenced by transforming growth factor beta 1 and heterotypic cell interactions. J Neuroimmunol 58: 101-110.

REN JQ, AIKA Y, HEIZMANN CW, KOSAKA T (1992) Quantitative analysis of neurons and glial cells in the rat somatosensory cortex with special reference to GABAergic neurons and parvalbumin-containing neurons. Exp Brain Res 92: 1-14

SAVCHENKO VL, MCKANNA JA, NIKONENKO IR, SKIBO GG (2000) Microglia and astrocytes in the adult rat brain: comparative immunocytochemical analysis demonstrates the efficacy of lipocortin 1 immunoreactivity. Neuroscience 96: 195-203.

STURROCK RR (1976) Light microscopic identification of immature glial cells in semithin sections of the developing mouse corpus callosum. J Anat 122: 521-537.

TKACHEV D, MIMMACK ML, RYAN MM, WAYLAND M, FREEMAN T, JONES PB, STARKEY M, WEBSTER MJ, YOLKEN RH, BAHN S (2003) Oligodendrocyte dysfunction in schizophrenia and bipolar disorder. Lancet 362: 798-805.

ZHOU M, SCHOOLS GP, KIMELBERG HK (2000) GFAP mRNA positive glia acutely isolated from rat hippocampus predominantly show complex current patterns. Brain Res Mol Brain Res 76: 121-131. 